\title{
ERRATUM
}

Ali Etebari · Pavlos P. Vlachos

\section{Improvements on the accuracy of derivative estimation from DPIV velocity measurements}

Published online: 20 December 2005

(C) Springer-Verlag 2005

\section{Experiments in Fluids (2005). DOI 10.1007/s00348- 005-0037-1}

In the original publication of this article the figure legends for Figs. 2, 3, 4, 5, 6, 7, 8, 9 were unfortunately omitted. We apologize for this mistake. The correct versions of Figs. 2, 3, 4, 5, 6, 7, 8, 9, with all parts included, can be seen here.
The online version of the original article can be found at http:// dx.doi.org/10.1007/s00348-005-0037-1

\footnotetext{
A. Etebari $(\varangle) \cdot$ P. P. Vlachos

Department of Mechanical Engineering, Virginia Tech,

215 Norris Hall, Blacksburg, VA 24061, USA

E-mail: aetebari@vt.edu

Tel.: + 1-540-2313366

E-mail: pvlachos@vt.edu
}

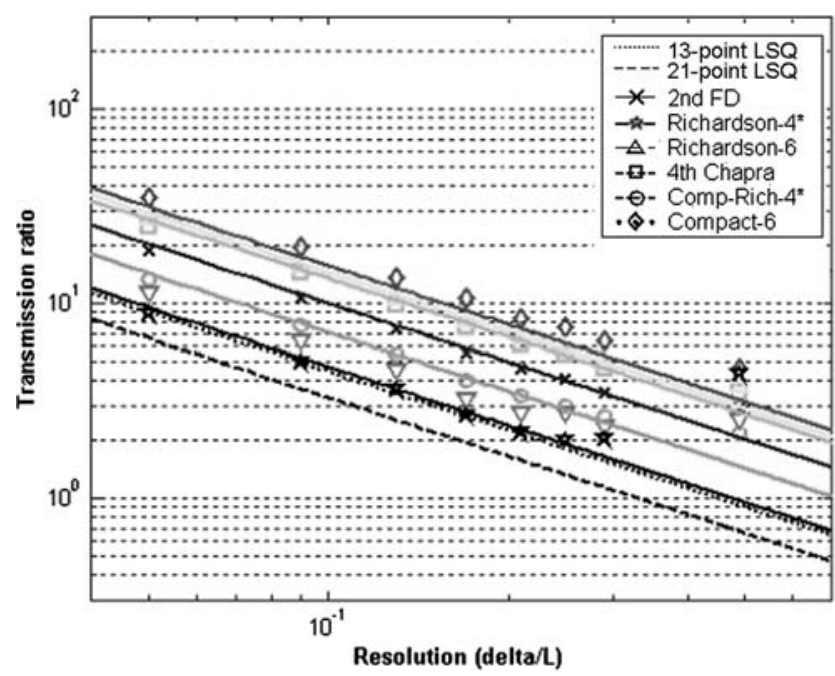

Fig. 2 Random error transmission ratio as a function of $\Delta / L$

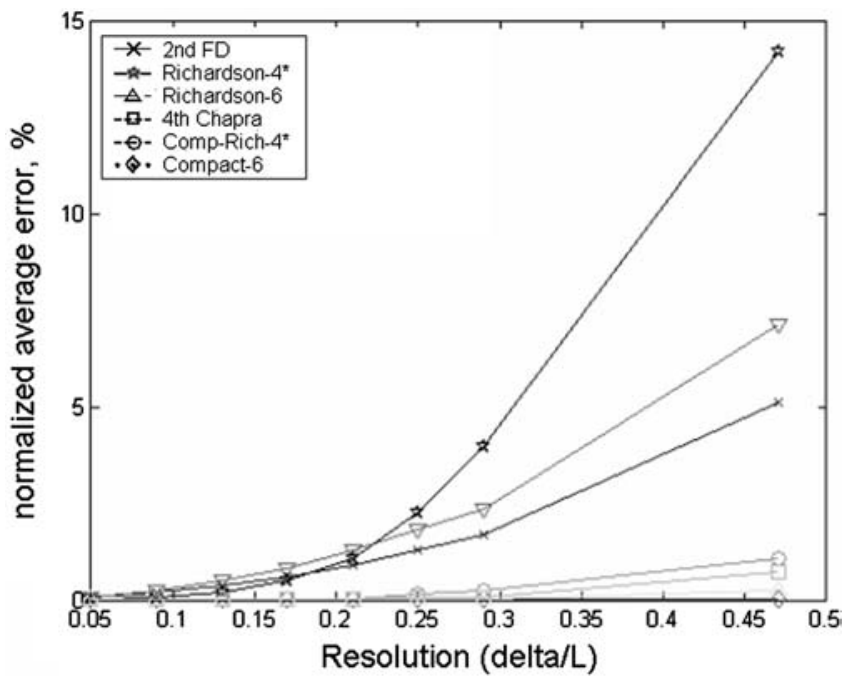

Fig. 3 Vorticity bias error (\%) as a function of spatial resolution 


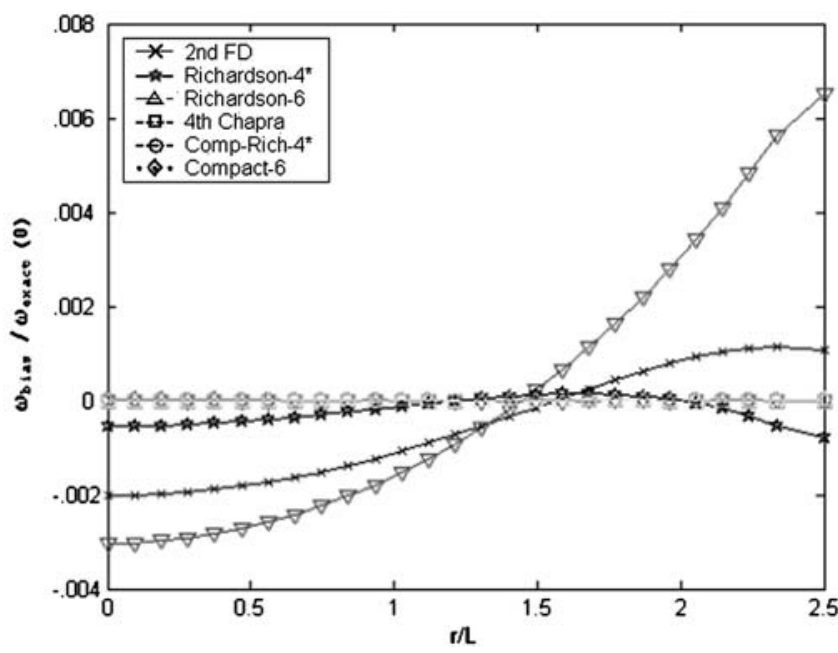

Fig. 4 Vorticity bias error $(\%)$ versus position $(\Delta / L=0.05)$

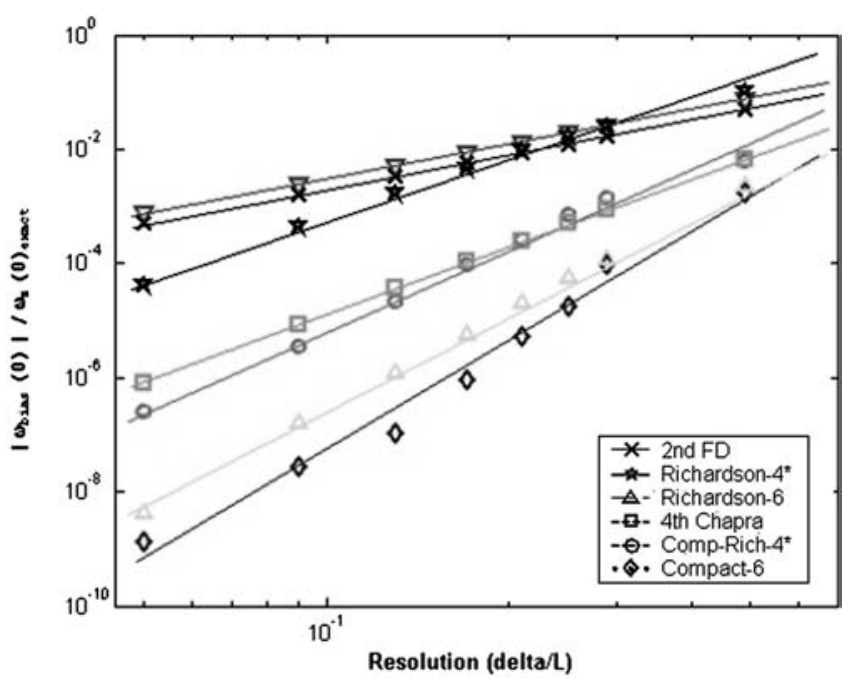

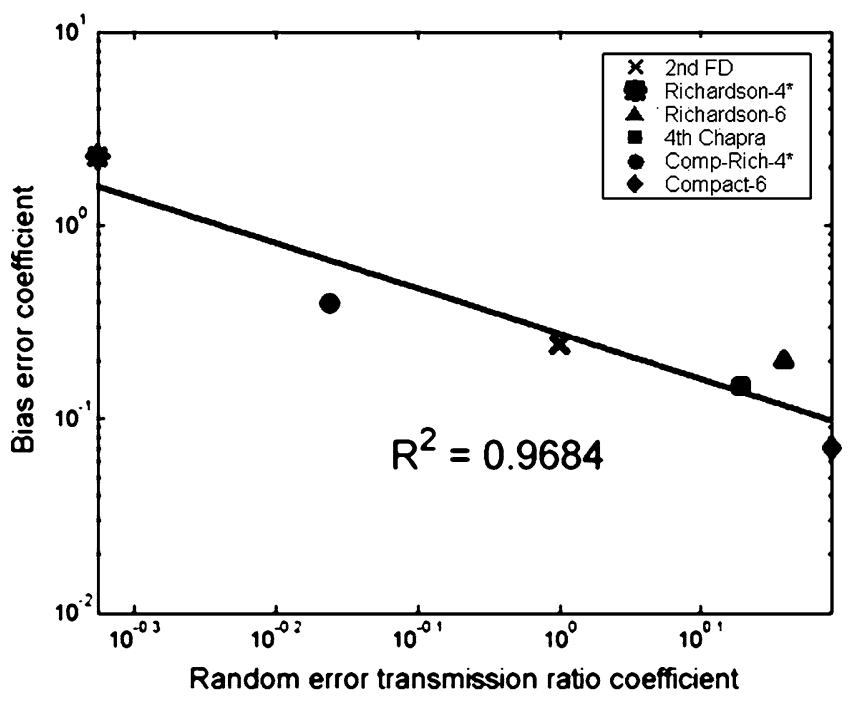

Fig. 6 Relationship between the coefficient of the power law and the transmission ratio

Fig. 5 Bias error at the vortex center versus $\Delta / L$

Fig. 7 Comparison of transfer functions for the FD2, comp-4, Rich-4, and CR4* schemes

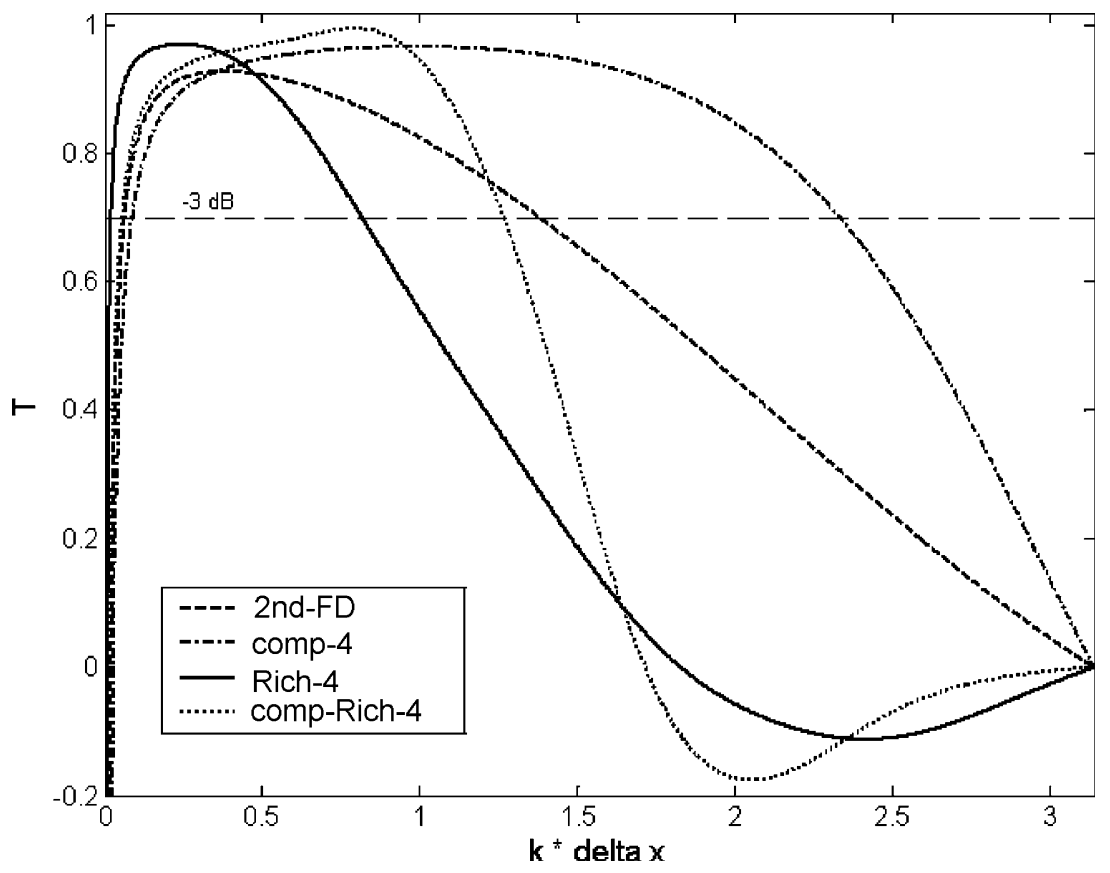




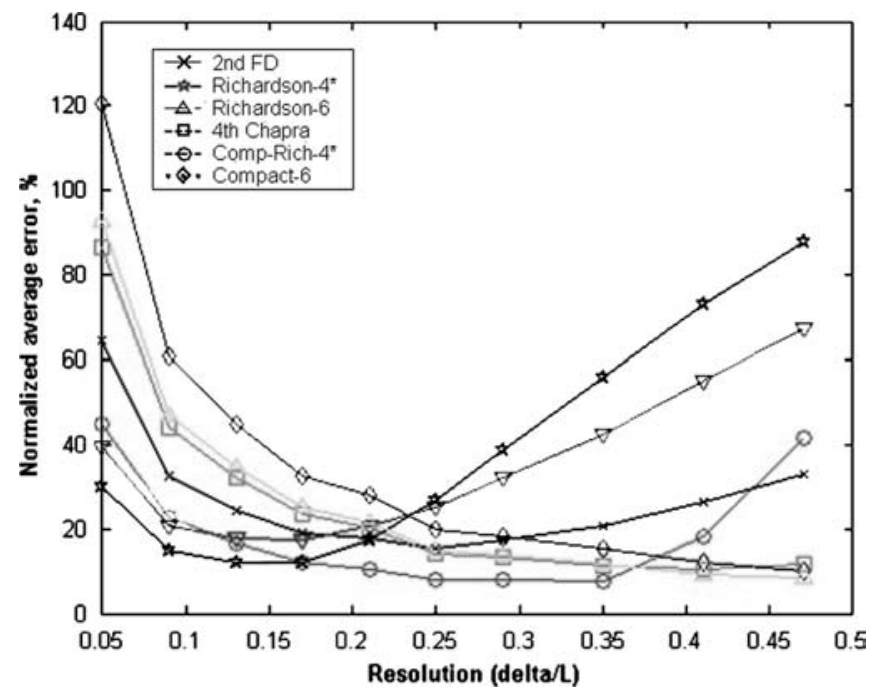

Fig. 8 Vorticity total error $(\%)$ as a function of spatial resolution for wave numbers $=1-7$

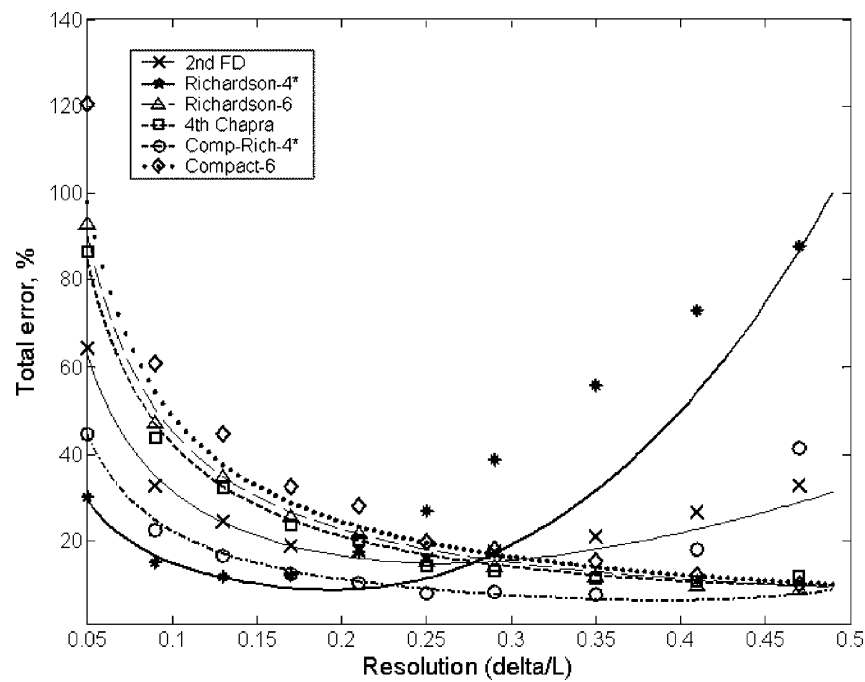

Fig. 9 Total error prediction for wave numbers $=1-7$ 